\title{
Spatial Variability of Photosynthetically Active Radiation in a Greenhouse
}

\author{
E.A. Guertal ${ }^{1}$ and C.B. Elkins ${ }^{2}$ \\ Department of Agronomy and Soils, Auburn University, Auburn, AL 36849
}

Additional index words. geostatistics, light, semivariogram, statistics

\begin{abstract}
Photosynthetically active radiation (PAR) was measured at two times of day (8:00 AM and noon Central Standard Time) in a $915 \times 915-\mathrm{cm}$ area of a $1006 \times 915-\mathrm{cm}$ gable roof greenhouse. PAR measurements were taken across a grid at 40-cm intervals, a total of 529 data points. Spatial variation of PAR in the greenhouse was evaluated through contour plots and the geostatistical technique of semivariogram construction. Semivariograms provide a visual guide to the degree of spatial correlation of a variable, allowing a quantification of the distance at which variables cease to be spatially correlated (the range) Measured PAR contained distinct zones of lowered values, a function of overhead greenhouse structures, wallhung electrical boxes, and tall plants in adjacent greenhouses. Although the amount of PAR changed over time, zones of high and low PAR remained relatively constant, except at the sides of the greenhouse. Constructed semivariograms revealed that PAR contained strong spatial correlation (up to a 350-cm separation) as measured in the north-south direction, moving parallel to greenhouse bench placement. When PAR measurements perpendicular to benches (eastwest) were used in directional semivariograms PAR was found to be completely random, plotting as a horizontal line called a nugget effect. Thus, plants placed perpendicular to the greenhouse benches (east-west) would not be affected by the spatial correlation of PAR.
\end{abstract}

Many greenhouse studies are conducted using the experimental design principles of blocking, randomization and replication. Such techniques are designed to remove variability that may underlie treatment effects (Bhatti et al., 1991; Cochran and Cox, 1957). Experiments arranged in such a manner are usually analyzed via classical techniques such as analysis of variance, regression analysis, or analysis of mean squares. Statistical models used in such analyses assume that the data is independent, with no correlation between data. However, if the data is positively correlated as a function of distance, and this correlation decreases between observation points with increasing distance, a confidence interval that is too narrow will be calculated if the presence of correlation is ignored (Cressie, 1993). Typically, the variance of a parameter with positive spatial correlation will be larger than the variance of that parameter when independence is assumed (Haining, 1988).

Many natural variables distributed in space do contain spatial correlation. Randomization and blocking may fail to eliminate the underlying spatial relationship (Bhatti et al., 1991; Stroup et al., 1994). Underlying spatial correlation in randomized and blocked field data has been demonstrated, especially in breeding trials (Kempton and Lockwood, 1984; Stroup et al., 1994) and fertility experiments (Bhatti et al., 1991).

The empirically derived Fairfield Smith law describes the relationship of variances among plots within a given experimental area. If yields from plots of a given size have a variance $V_{1}$, then yields per unit area derived from plots of area $x$ will have a variance $\mathrm{V}_{x}$ such that $\mathrm{V}_{x}=\mathrm{V}_{1} / \mathrm{x}^{b}$, where $b$ is some constant between 0 and 1 (Pearce, 1976; Smith, 1938). The size of $b$ is a measure of similarity between plots; plots that are highly correlated have a $b$ value close to 0 , a value close to 1 indicates limited correlation (Binns, 1982). An average $b$ value of 0.25 was found in a reanalysis of 44 field experiments (Ferguson, 1962); average $b$ values of 0.41 to 0.48 have been observed in greenhouse experiments (Alvey, 1961).

Received for publication 13 Apr. 1995. Accepted for publication 14 Nov. 1995. Contribution of the Dept. of Agronomy and Soils, Auburn Univ., and the Alabama Agricultural Experiment Station. The cost of publishing this paper was defrayed in part by the payment of page charges. Under postal regulations, this paper therefore must be hereby marked advertisement solely to indicate this fact.

${ }^{1}$ Assistant professor.

${ }^{2}$ Superintendent, Plant Growth Facilities.
In the early 1960s a new type of statistical procedure for examining spatial correlation, called geostatistics, was introduced. Geostatistics encompasses theory and methods for describing and modeling spatial data. Promoted and used extensively since the early 1960s, geostatistics was first developed for mineral mining (Matheron, 1963), and has since been adopted by many other disciplines, including agriculture (Trangmar et al., 1985; Unlu et al., 1990). Unlike conventional statistical methods, geostatistics uses the locations of data as part of the analytical method. In addition, geostatistics assumes that data more proximate to one another will be more highly correlated than data that are most distantly separated. Specifically, spatial data are described as a function of both the distance and direction of the separations between data locations.

A major component of a geostatistical analysis is the construction of experimental semivariograms. Experimental semivariograms use empirical data to provide a picture of possible spatial correlation that exists within a data set. If spatial correlation is present, classical statistical methods based on uncorrelated data may provide biased tests of significance when used to model the treatment response.

Semivariogram construction utilizes data collected from an area of interest to produce a curve that describes the spatial variability for a single response variable. The equation for semivariogram construction (Cressie, 1993) is

$$
\gamma(h)=\frac{1}{2 N(h)} \sum_{N(h)}\left[Z\left(s_{i}\right)-Z\left(s_{j}\right)\right]^{2}
$$

where $\gamma(h)=$ semivariogram value at lag distance $\mathrm{h}, N(h)=$ number of sample pairs at lag distance $h$, and $Z(s)=$ samples $i$ and $j$ separated by the lag distance $h$.

The lag distance is the separation distance between samples, usually a function of the grid spacing over which samples were collected. The calculated semivariogram value at each lag distance, $h$, is plotted against that lag distance, and a semivariogram is created.

Figure 1 illustrates an experimental semivariogram. The plotted points initially increase, then plateau at a value called the sill of the semivariogram plot. The lag distance where the sill is first attained is called the range of the spatial correlation of the data. The distance from 0 to the $y$-intercept is the nugget effect, that portion of the semivariance $[\gamma(h)]$ due to random variability. The initial 


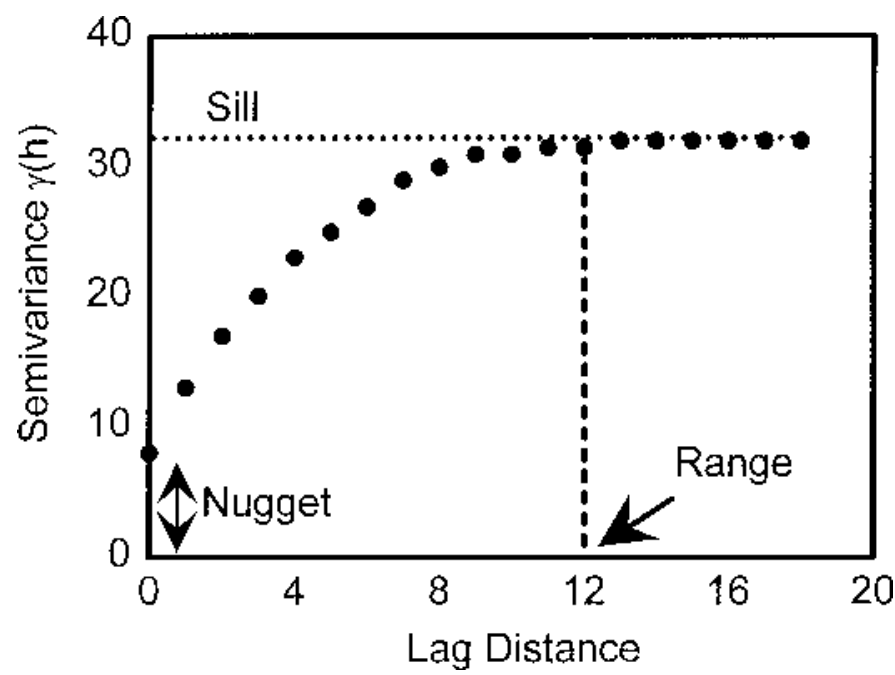

Fig. 1. Example semivariogram.

increase in semivariogram values (up to the sill) occurs over the area where values are spatially correlated. If the semivariogram does not increase but instead plots as a horizontal band of points the spatial correlations are weak (a complete nugget effect), and the data can therefore be considered to be uncorrelated. Theoretically, the existence of a complete nugget effect is the only situation where classical statistical methods may be used (Vieira et al., 1983).

Research which explores the spatial variability of soil properties is well-documented, including characteristics such as percent sand, silt and clay (Campbell, 1978; Ovalles and Collins, 1988; Pierson and Mulla, 1990); and hydraulic conductivity and aggregate stability (Unlu et al., 1990; Mulla, 1988). The spatial variability of soil chemical properties such as $\mathrm{pH}$, nitrogen and phosphorus have also been analyzed (West et al., 1989; Wollenhaupt et al., 1994).

Given that the spatial variability of soil properties in crop production systems has been documented, it was our interest to explore the spatial variability of variables that affect greenhouse production systems. In the same way that spatial correlations of a soil nutrient or physical property might affect field studies, we chose to examine the spatial variability of photosynthetically active radiation (PAR) in a research greenhouse. PAR was chosen because it affects plant growth and is relatively easy and quick to measure. Structural members, installed equipment and tall vegetation in adjacent houses create shadows in greenhouses, producing variation in solar radiation intercepted by plants that will affect treatment response. Quantification of solar radiation variation is essential for the proper design, conduct and interpretation of greenhouse experiments. The objectives of this study were to 1)

Table 1. Descriptive statistics for PAR measured in greenhouse at 8:00 AM and noon CST.

\begin{tabular}{lrcccc}
\hline \hline Time & Min & Max & Mean & SD & Skewness \\
\hline \multicolumn{5}{c}{$\mu \mathrm{mol} \cdot \mathrm{m}^{-2} \cdot \mathrm{s}^{-1}$} \\
8:00 AM & 89.4 & 1166.3 & 470.9 & 316.8 & 0.68 \\
Noon & 109.0 & 1548.9 & 812.4 & 534.6 & -0.10 \\
Average $^{\mathrm{z}}$ & 99.2 & 1245.3 & 641.7 & 333.3 & 0.008 \\
\hline
\end{tabular}

z8:00 AM plus noon)/2. assess patterns of spatial variability in PAR in a research greenhouse at two sampling times, 2) construct experimental semivariograms from collected PAR data, and 3) determine if constructed semivariograms provide information about the degree of spatial correlation of PAR present in a greenhouse.

\section{Materials and Methods}

Data collection. PAR measurements were made at $40-\mathrm{cm}$ intervals in a $915 \times 915-\mathrm{cm}$ zone of a north-south oriented gable roof greenhouse of conventional aluminum frame and glass construction at the Plant Science Research Center at Auburn, Ala. (lat 32 36' long 85 29'). PAR was measured by manually reading the current output from a LI-COR quantum light sensor with a Metex M-4650 digital multimeter, in units of $\mu \mathrm{mol} \cdot \mathrm{m}^{-2} \cdot \mathrm{s}^{-1}$. The sensor was mounted horizontally in a light-weight plastic holder that was supported by and equipped to slide along two Kevlar braided fishing lines. Kevlar line was selected because it has a low stretch coefficient, a high tensile strength and is light-weight. The two lines, spaced $4 \mathrm{~cm}$ apart, were attached to metal brackets that clipped onto metal pipes mounted $1.3 \mathrm{~m}$ above the greenhouse floor along the sides of the greenhouse zone. Tension was applied to the support lines by rotating one of the metal brackets around the pipe and clamping the bracket in place. A third line stretched parallel to the two support lines was marked at 40-cm intervals as an index for positioning the light sensor. The pipes were marked at $40 \mathrm{~cm}$ intervals as an index for positioning the support line brackets. The support line brackets were clamped in place on the pipes at the first set of marks and the sensor holder was moved along the lines and a reading was taken at each mark. The support lines were advanced along the pipes to the next marks and the procedure was repeated until 529 readings were recorded.

Readings were taken on 10 Mar. 1995, a day selected because of absence of any cloud cover. Readings were collected at 8:00 AM and noon Central Standard Time. Each set of 529 readings took about $45 \mathrm{~min}$ to record, so that actual data collection times occurred from 8:00 to 8:45 AM and 11:45 to 12:30 PM. No attempts were made to remove plants from neighboring greenhouses or move humidity

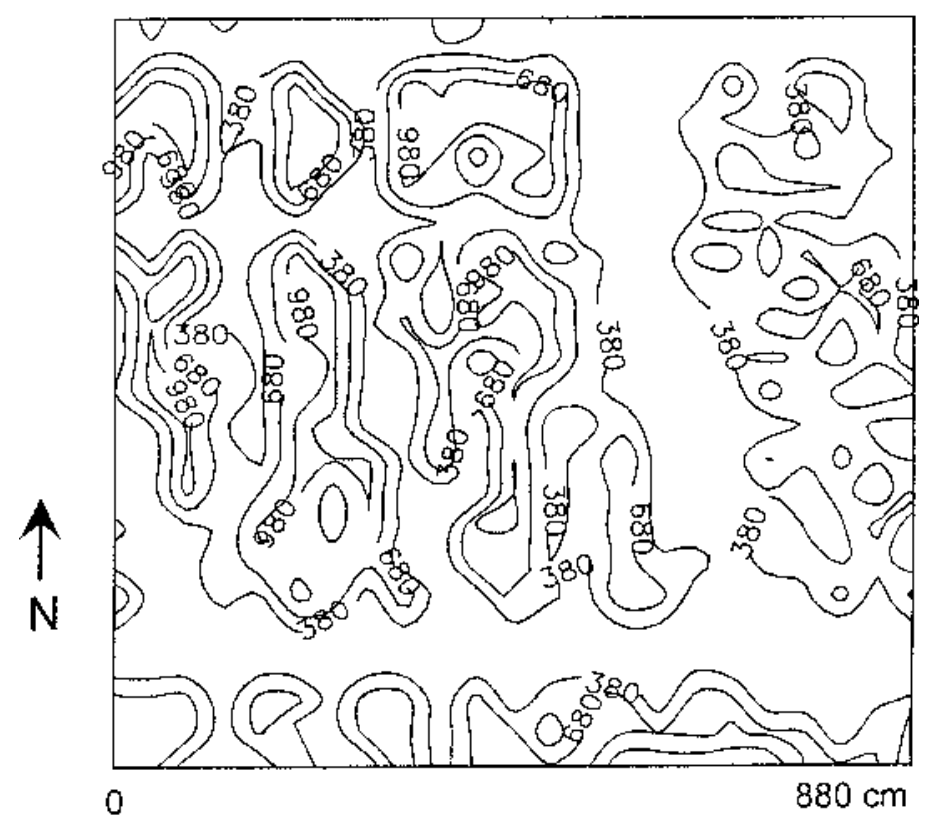

Fig. 2. Contour plot of PAR in the greenhouse measured at 8:00 AM CST. 


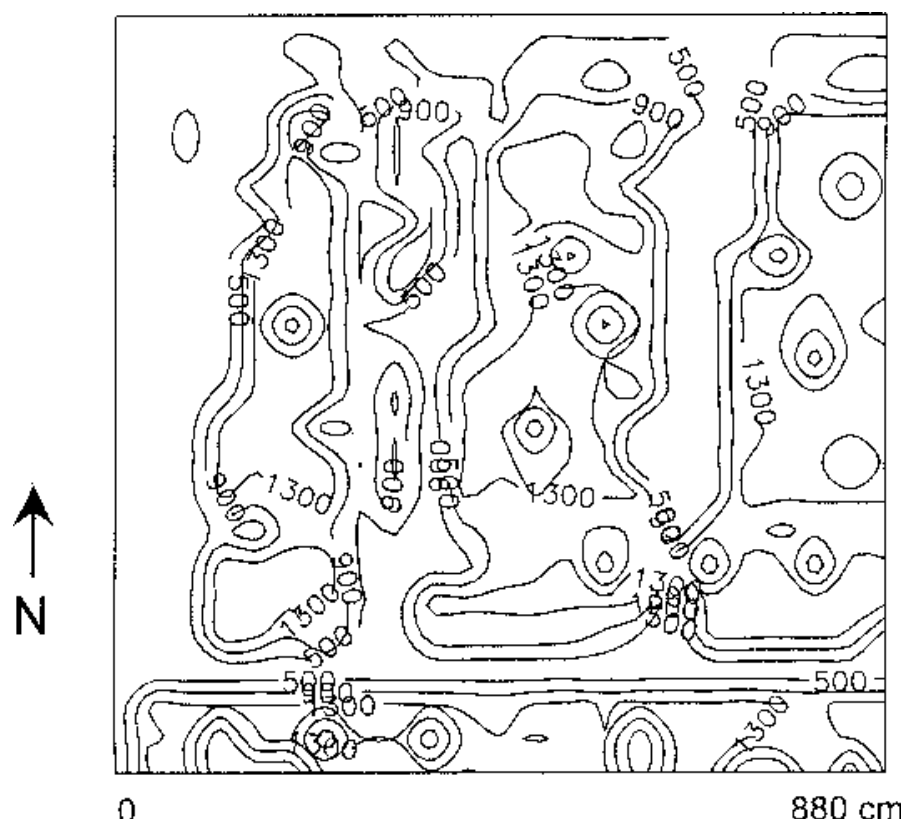

Fig. 3. Contour plot of PAR in the greenhouse measured at noon CST.

sensors or other permanent fixtures in the greenhouse.

Statistical manipulation. All data were initially analyzed by standard statistical methods, providing estimates of the mean, maximum, minimum, standard deviation and skew for each sampling time. All geostatistical analyses were performed using the GEOEAS (v 1.1) geostatistical package (Englund and Sparks, 1988). Spatial relationships of greenhouse PAR were described through the use of omnidirectional and directional semivariograms, employing actual data values.

Omnidirectional semivariograms were calculated from every pair of data points (at $40 \mathrm{~cm}$ increments) within one-half of the total sampled distance, considering every direction at each selected lag distance. Directional semivariograms were calculated over two directions: east and north, each with an angular tolerance of $22.5^{\circ}$. At a given lag distance all points located within $22.5^{\circ}$ of the selected direction were included in the semivariogram calculation, again limited to a final lag length of one-half the total sampled distance. The two directional semivariograms provided an indication of spatial variation of PAR moving perpendicular (east) and parallel (north) to plant benches in the greenhouse.

Directional semivariograms were used to determine if anisotropy existed within the data set. Anisotropies are said to occur if semivariograms change over varying directions, and are indicated by different sills or ranges in the experimental semivariograms, or both.

\section{Results}

Table 1 lists the mean, maximum, minimum, standard deviation and skew of the 8:00 AM, noon, and average PAR data. Probability plots of the data (not shown) indicate that the data was normally distributed. The data appeared to contain a bimodal distribution centered around groups of low and high values, a finding supported by large standard deviations and small skewness in the data.

Contour plots of PAR readings indicated distinct zones of reduced PAR within the greenhouse, a result of overhead structures, wall-hung electrical equipment or tall plants in adjacent houses (Figs. 2 and 3). Tall plants growing in the greenhouse directly to the east of the greenhouse of interest created reductions in PAR that were strongly evident at 8:00 AM. Noon readings indicated that electrical circuit boxes mounted outside the greenhouse on the west wall created reductions in PAR in that side of the greenhouse. Zones that continually produced lower PAR readings were those areas underlying a structural beam at the top of the greenhouse (a zone running directly east-west located at about 40 $\mathrm{cm}$ north) and two translucent plastic air ducts hanging $3.5 \mathrm{~m}$ from the ceiling, plus a ceiling-mounted folded shade cloth (two zones running north-south spaced at about 350 and $650 \mathrm{~cm}$ east).

Omnidirectional semivariograms (Fig. 4) for 8:00 AM and noon indicate that PAR contains strong spatial correlation throughout the greenhouse. Both sets of data contained small nugget effects and well-defined ranges. The omnidirectional PAR semivariogram created from data collected at noon revealed a shape indicative of a trend, as the sill did not level, and behavior near the origin may have been hyperbolic. In this case, behavior near the origin is difficult to assess because there were no sampling distances closer than $40 \mathrm{~cm}$, so inferences about spatial behavior of PAR closer than $40 \mathrm{~cm}$ cannot be made. The term trend is used here to indicate the presence of a large-scale variation in the data, possibly a result of the sun's change in position during the 45-min measurement time. Changing position of the sun from 8:00 to 8:45 AM, which occurred at a lower angle in the sky, did not create the same pronounced trend in the data.

Omnidirectional semivariograms revealed that PAR measurements closer than 100 and $225 \mathrm{~cm}$ were spatially correlated, for the 8:00 AM and noon measurements, respectively.

Because pot placement in greenhouse experiments is partially a function of bench location, directional semivariograms were
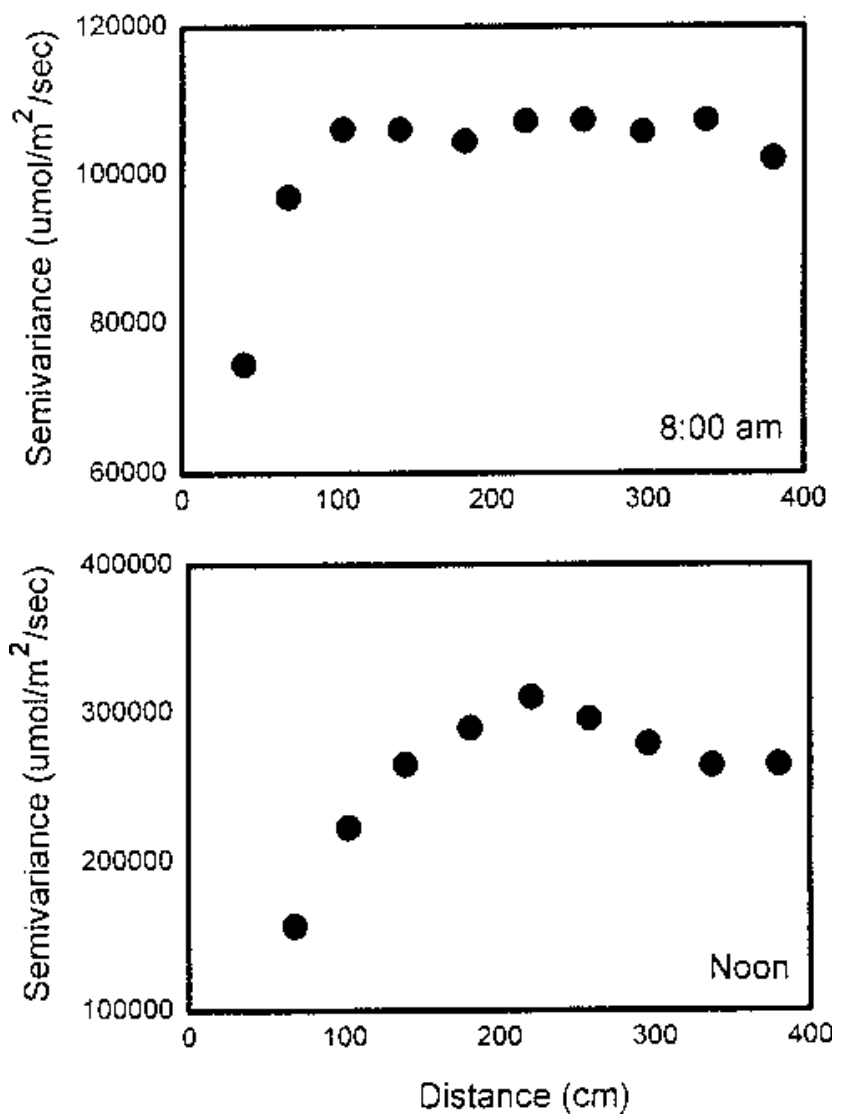

Fig. 4. Omnidirectional semivariograms of PAR at 8:00 AM and noon CST. 

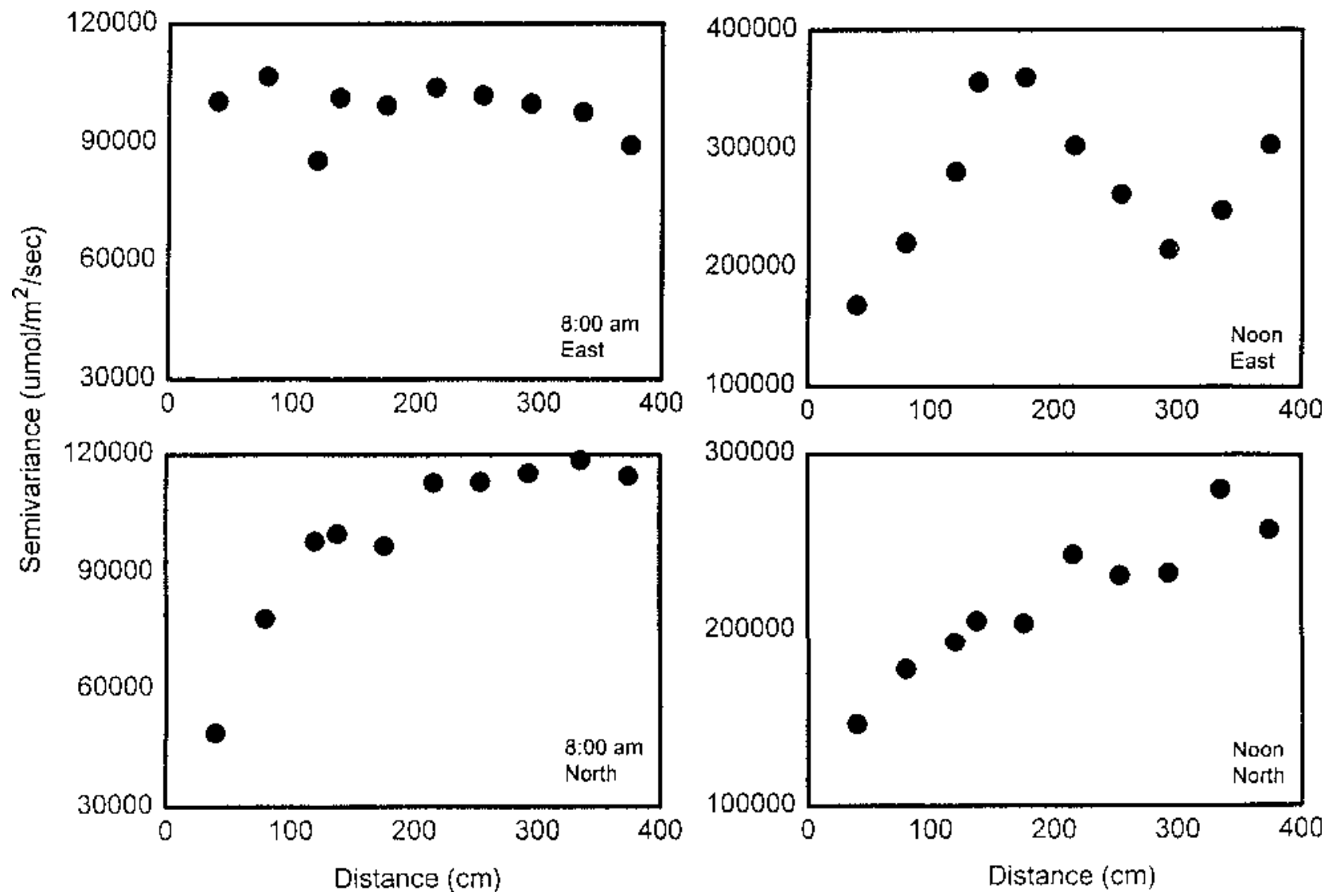

Fig. 5. Directional semivariograms of PAR at 8:00 AM and noon CST.

created to observe spatial correlation of PAR perpendicular and parallel to greenhouse benches. Directional semivariograms (Fig. 5) exhibited pronounced anisotropies, as directional semivariograms from PAR data parallel to benches (direction north) contained strong spatial correlation, while directional semivariograms of PAR data perpendicular to the benches exhibited a completely random, or nugget effect.

The presence of trend in the noon omnidirectional semivariogram was confirmed in the noon east directional semivariogram. Appearance of trend solely in the east-west direction is likely, as the sun would move in that direction during the measurement period. When trend was removed from the noon east directional semivariogram using a median polish technique (Cressie, 1993), the directional semivariogram resemble a completely random nugget effect, similar to the 8:00 AM east directional semivariogram (Fig. 6).

\section{Discussion}

PAR, as measured in one greenhouse, contained strong spatial correlation in one direction (north-south) of that greenhouse. North directional semivariograms of PAR data from 8:00 AM and noon readings indicate that $\mathrm{PAR}$ is spatially correlated to a distance of about $350 \mathrm{~cm}$. Once past that distance, PAR, measured parallel to the plant benches, would be a completely random effect. PAR measured perpendicular to the plant benches was always a completely random effect, as shown by semivariograms with a complete nugget effect.

PAR measured in this experiment was a point measurement, and further studies are needed to study the cumulative spatial variability of PAR. An examination of the average PAR (average of 8:00 AM plus noon) revealed spatial behavior that was similar to that observed at 8:00 AM and noon; averaging the values did not mediate the spatial variability of PAR within the greenhouse.

The effect of spatially correlated PAR on specific treatment effects or plant response was not a part of this study. Plant response would not likely be linear with change in PAR; large changes in PAR across the greenhouse may not create the same large differences in plant response. However, it is likely that differences in PAR would affect some components of an experiment, including plant height, soil or potting media moisture, plant dry matter

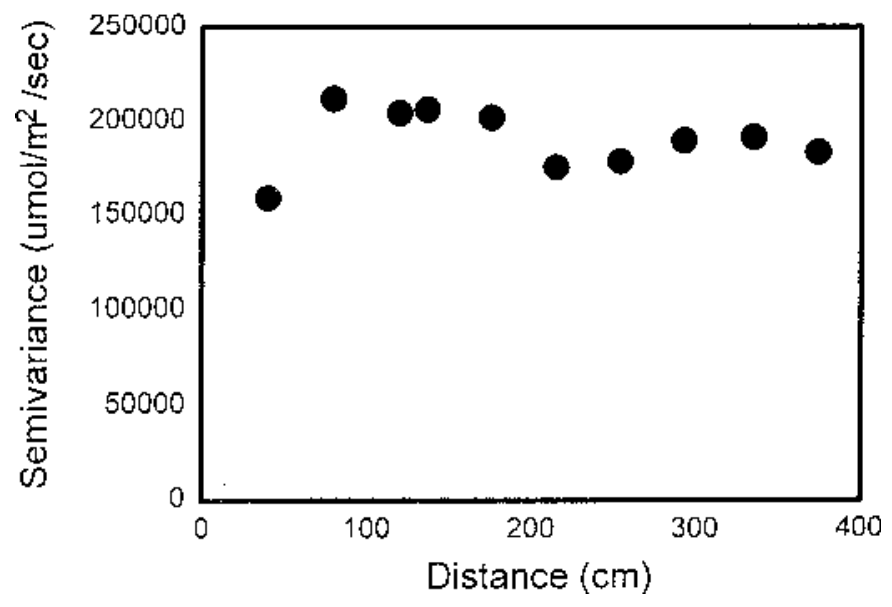

Fig. 6. East directional semivariogram of PAR at noon CST with trend removed. 
accumulation, and blooming date. In this greenhouse, treatments placed closer together than $350 \mathrm{~cm}$ (running north-south) would not receive PAR as a completely random effect (at least at any specific time of day), and spatial correlation of PAR would underlie treatment effects. If treatments were placed perpendicular to the bench (running east-west) PAR would be a completely random effect.

In this specific case, to avoid the underlying affect of spatial correlation of PAR, a greenhouse experiment arranged as a randomized complete block design would best be served by placing treatments within a block perpendicular to the greenhouse bench (east-west), and blocks should be placed either on adjacent benches or separated by $350 \mathrm{~cm}$ on the same bench. Alternatively, using a completely randomized design is frequently recommended when environmental variation is not regular. The use of spatial statistical techniques such as presented here demonstrate that spatial variability in a greenhouse is a real and changing phenomena, and the presence of such variability should be considered when initiating a greenhouse experiment.

\section{Literature Cited}

Alvey, N.G. 1961. Methods likely to reduce the glasshouse area needed for experiments with the tomato. Plant and Soil 14:22-32.

Bhatti, A.U., D.J. Mulla, F.E. Koehler, and A.H. Ghurmani. 1991. Identifying and removing spatial correlation from yield experiments. Soil Sci. Soc. Amer. J. 55:1523-1528.

Binns, M.R. 1982. The choice of plot size in randomized block experiments. J. Amer. Soc. Hort. Soc. 107:17-19.

Campbell, J.B. 1978. Spatial variation of sand content and $\mathrm{pH}$ within single contiguous delineations of two soil mapping units. Soil Sci. Soc. Amer. J. 42:460-464.

Cochran, W.G. and C. Cox. 1957. Experimental designs. 2nd ed. Wiley, New York.

Cressie, N.A.C. 1993. Statistics for spatial data. Wiley, New York.

Englund, E. and A. Sparks. 1988. GEO-EAS. Geostatistical environmental assessment software user's guide. USEPA, Las Vegas, Nev.
Ferguson, J.H.A. 1962. Random variability in horticultural experiments. Euphytica 11:213-220.

Haining, R.P. 1988. Estimating spatial means with an application to remotely sensed data. Comm. in Statistics. Theory and Methods. 17:573597.

Kempton, R.A. and G. Lockwood. 1984. Inter-plot competition in variety trials of field beans (Vicia faba L.) J. Agr. Sci. (Cambridge) 103:293302.

Matheron, G. 1963. Principles of geostatistics. Econ. Geolo. 58:12461266.

Mulla, D.J. 1988. Estimating spatial patterns in water content, matric suction and hydraulic conductivity. Soil Sci. Soc. Amer. J. 52:15471553.

Ovalles, F.A. and M.E. Collins. 1988. Evaluation of soil variability in northwest Florida using geostatistics. Soil Sci. Soc. Amer. J. 52:17021708.

Pearce, S.C. 1976. An examination of Fairfield Smith's law of environmental variation. J. Agr. Sci. 87:21-24.

Pierson, F.B. and D.J. Mulla. 1990. Aggregate stability in the Palouse region of Washington: Effect of landscape position. Soil Sci. Soc. Amer. J. 54:1407-1412.

Smith, H. Fairfield. 1938. An empirical law describing heterogeneity in the yields of agricultural crops. J. Agr. Res. 28:1-23.

Stroup, W.W., P.S. Baenziger, and D.K. Mulitze. 1994. Removing spatial variation from wheat yield trials: A comparison of methods. Crop Sci. 86:62-66.

Trangmar, B.B., R.S. Yost, and G. Uehara. 1985. Application of geostatistics to spatial studies of soil properties. Adv. Agron. 38:45-91.

Unlu, K., D.R. Nielsen, J.W. Biggar, and F. Morkoc. 1990. Statistical parameters characterizing the spatial variability of selected soil hydraulic properties. Soil Sci. Soc. Amer. J. 54:1537-1547.

Vieira, S.R., J.L. Hatfield, D.R. Nielsen and J.W. Biggar. 1983. Geostatistical theory and application to variability of some agronomical properties. Hilgardia 51:1-75.

West, C.P., A.P. Mallarino, W.F. Wedin, and D.B. Marx. 1989. Spatial variability of soil chemical properties in grazed pastures. Soil Sci. Soc. Amer. J. 53:784-789.

Wollenhaupt, N.C., R.P. Wolkowski, and M.K. Clayton. 1994. Mapping soil test phosphorus and potassium for variable-rate fertilizer application. J. Prod. Agr. 7:441-448. 